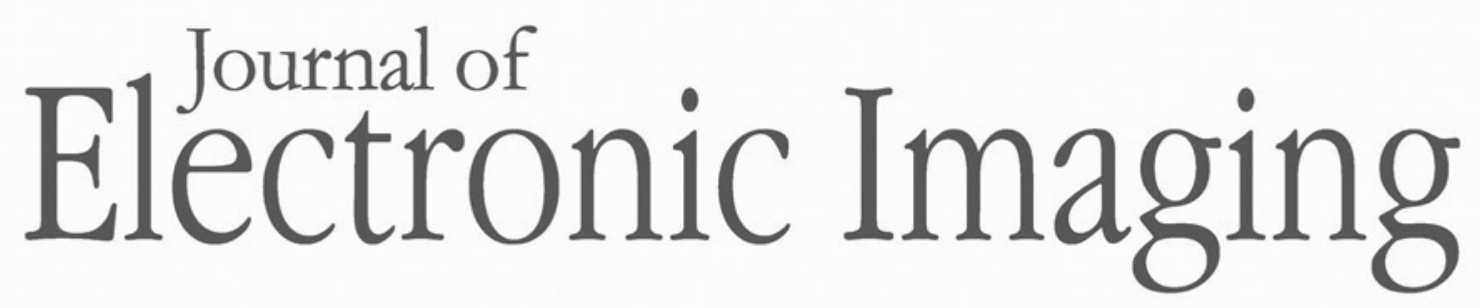

JElectronicImaging.org

\title{
Top Reviewers of 2015
}

Gaurav Sharma

Karen Egiazarian 


\section{Top Reviewers of 2015}

During 2015 more than 700 papers were submitted for potential publication in the Journal of Electronic Imaging. More than 900 reviewers from nearly 60 countries were involved in the evaluation process.

We would like to thank all of them together with the members of the editorial board of the Journal of Electronic Imaging for their extremely important work and invaluable assistance in the reviewing process to secure a high level of scientific publication.

Twenty reviewers completed reviews for six or more papers. To choose the best reviewers among them we've used both qualitative (ranking average for all submissions) and quantitative (number of reviews completed, average days to perform a review, and percentage of reviews on time) criteria. Based on those, the top five reviewers for the Journal of Electronic Imaging for the year 2015 have been selected. They are listed below in alphabetical order:

- Chenguang Liu, Utah State University, USA

- Andy Jinhua Ma, Johns Hopkins University, USA

- Ayan Seal, Jadavpur University, India

- Yi Zhang, University of Dayton, USA

- Dexing Zhong, Xi'an Jiaotong University, China

\section{Gaurav Sharma Outgoing Editor-in-Chief}

Karen Egiazarian Incoming Editor-in-Chief 